\title{
Glauber gluons in pion-induced Drell-Yan processes
}

\author{
Hsiang-nan Li* \\ Institute of Physics, Academia Sinica, Nankang, Taipei 115, Taiwan, Republic of China \\ E-mail: hnliephys.sinica.edu.tw
}

We point out that the existence of Glauber gluons in the $k_{T}$ factorization theorem can account for the violation of the Lam-Tung relation, namely, the anomalous lepton angular distribution observed in pion-induced Drell-Yan processes. This mechanism is compared to other resolutions in the literature by means of vacuum effects and Boer-Mulders functions. We propose to discriminate the above resolutions by measuring the $p \bar{p}$ Drell-Yan process at GSI and J-PARC.

XV International Conference on Hadron Spectroscopy-Hadron 2013

4-8 November 2013

Nara, Japan

${ }^{*}$ Speaker. 


\section{Introduction}

Though a Drell-Yan process has been considered as being fully understood, it reveals a puzzling behavior since 80 's. The angular distribution of produced lepton pairs is expressed in the Collins-Soper frame as

$$
\frac{1}{\sigma} \frac{d \sigma}{d \Omega}=\frac{3}{4 \pi} \frac{1}{\lambda+3}\left(1+\lambda c_{\theta}^{2}+\mu s_{2 \theta} c_{\phi}+v \frac{s_{\theta}^{2}}{2} c_{2 \phi}\right),
$$

with the notations $c_{\theta} \equiv \cos \theta$ and $s_{\theta} \equiv \sin \theta$. In the above expression $\theta$ is the angle between one of the leptons and the $z$ axis, and $\phi$ is the angle between the plane formed by one of the lepton momenta and the $z$ axis and the plane formed by the colliding beam momenta. The involved coefficients obey the Lam-Tung relation $1-\lambda-2 v=0$ [四], which holds under perturbative corrections

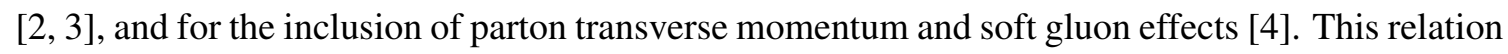
has been experimentally verified in the $p p$ and $p d$ Drell-Yan processes [[5]. However, significant violation was observed in pion-induced ones, which was found to increase with the lepton-pair transverse momentum $q_{T}$ [ [6, प, 四].

Resolutions of the above violation include the vacuum effect [Q, [0] that causes the transversespin correlation between colliding partons, and the Boer-Mulders (BM) functions [四], that introduce the spin-transverse-momentum correlation of a parton. The vacuum effect is flavor-blind [ए]], so it demands more effort to differentiate the $\pi p$ and $p p$ Drell-Yan processes. The BM proposal can differentiate the pion from the proton, because the involved anti-quark is a valence (sea) parton in the former (latter). In this talk we shall present a resolution by means of Glauber gluons in the $k_{T}$ factorization theorem [12, [13], whose effect might be significant due to the unique role of the pion as a Nambu-Goldstone (NG) boson and a $q \bar{q}$ bound state simultaneously [14]]. The emission of a final-state parton, that balances the lepton-pair transverse momentum, causes the required spintransverse-momentum correlation in the Glauber-gluon background. A simple discrimination is to measure the $p \bar{p}$ Drell-Yan process, which will exhibit strong violation of the Lam-Tung relation according to BM, but will not according to the uniqueness of the pion.

\section{Glauber phase}

It has been known that the $k_{T}$ factorization of complicated QCD processes involving more than two hadrons, such as hadron hadroproduction at the transverse momentum $q_{T}$ [ए2], [3]], is broken by residual infrared divergences from the Glauber region. It was then demonstrated that these Glauber divergences can be factorized into a new universal nonperturbative phase factor at low $q_{T}$, where the eikonal approximation holds even in the Glauber region [[15]. It is obvious that the $q_{T}$ spectra of $\lambda, \mu$ and $v$ meet the criteria for introducing the Glauber phase: since the low- $q_{T}$ spectra are concerned, the $k_{T}$ factorization is an appropriate theoretical framework; a final-state parton is required to balance the lepton-pair $q_{T}$, so at least three partons are involved; the Glauber gluons exist and are factorizable at low $q_{T}$, leading to the Glauber phase factor.

The leading-order diagrams for the parton-level scattering $\bar{q}\left(p_{1}\right)+q\left(p_{2}\right) \rightarrow \ell^{-}\left(k_{1}\right)+\ell^{+}\left(k_{2}\right)+$ $g\left(k_{3}\right)$ in the pion-proton Drell-Yan process are displayed in Fig. W. The diagrams with the gluon of momentum $k_{3}$ and the lepton pair being exchanged is implicit. The anti-quark (quark) carrying 


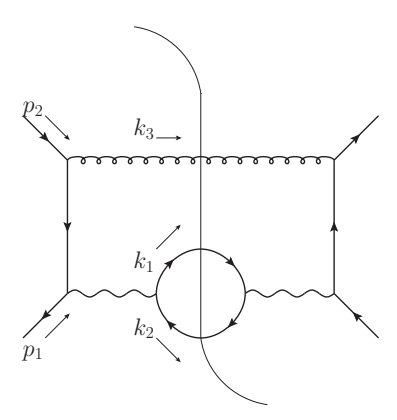

(a)

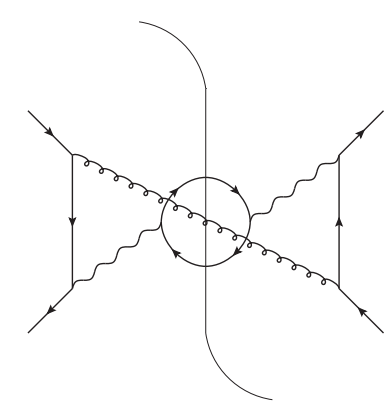

(b)

Figure 1: Some LO diagrams for lepton-pair production with $q_{T}$ in a Drell-Yan process.

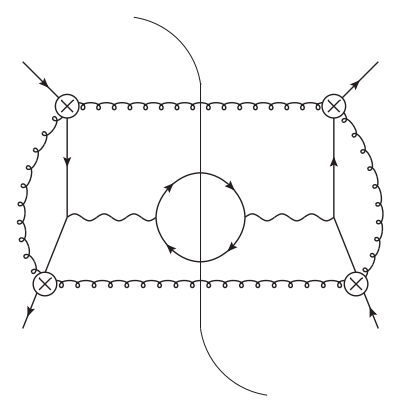

(a)

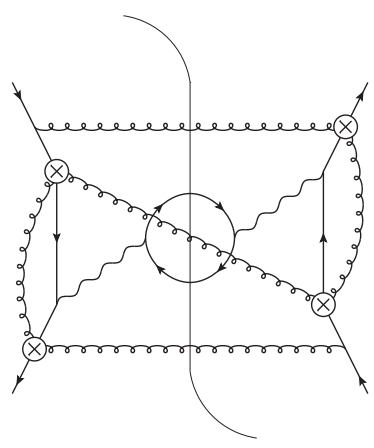

(b)

Figure 2: Factorization of Glauber gluons (vertical lines).

the momentum $p_{1}$ in the plus direction ( $p_{2}$ in the minus direction) is a parton of the pion (proton). The Glauber divergence arises from a higher-order correction, where a loop gluon of momentum $l$ connects, for example, a rung gluon exchanged between the two partons in the pion, and the lines in the upper half of Fig. W(a). The factorization of a Glauber gluon can be achieved at low $q_{T}$ by applying the eikonal approximation to the lines it connects, and by applying the Ward identity to all the attachments [15]. The outcome is displayed in Fig. D(a), where the upper and lower vertices denoted by $\otimes$ represent the functions $\delta\left(l^{+}\right)$and $\delta\left(l^{-}\right)$, respectively. Repeating the above procedures, we factorize the Glauber gluons in Fig. $\mathbb{Z}(\mathrm{b})$. Comparing Fig. $\mathbb{\nabla}(\mathrm{b})$ with $\mathbb{\nabla}(\mathrm{a})$, the existence of the Glauber divergence in the former demands one more rung gluon. That is, it is down by a power of $\alpha_{s}$, and regarded as a next-to-leading logarithm. Exponentiating the imaginary logarithms in Fig. 凹, we obtain the Glauber phase factor $\exp \left(i S_{e}\right)$ [प5]].

The uniqueness of the pion is hinted by numerous anomalous data involving pions in addition to the deviation of the Lam-Tung relation: the observed $B^{0} \rightarrow \pi^{0} \pi^{0}, \pi^{0} \rho^{0}$, and $\rho^{0} \rho^{0}$ branching ratios are 6 times of, 3 times of, and consistent with the perturbative QCD predictions [ए6]. It is not understood why the deviation becomes more significant as number of pions increases. This is the so-called $B \rightarrow \pi \pi$ puzzle. The $q_{T}$ spectrum of the pion hadroproduction is dramatically distinct from the hadroproduction of other hadrons: fitting to the data from $p p$ collisions at RHIC and LHC, 

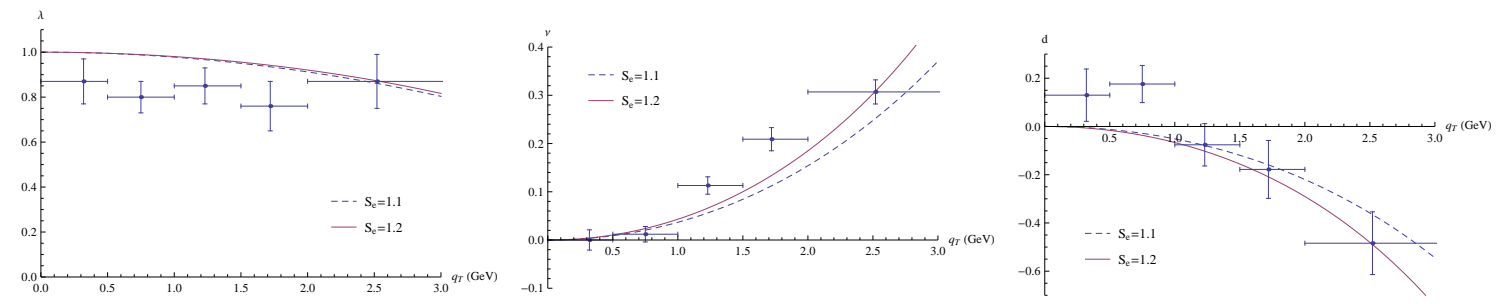

Figure 3: $q_{T}$ dependence of $\lambda, v$ and $1-\lambda-2 v$ for $S_{e}=1.1$ (dashed lines) and $S_{e}=1.2$ (solid lines).

and from $A u A u$ collisions with different centralities at RHIC implies that the former follows an exponential law, but the latter follows a power law [ㅍ]]. Nevertheless, the power-law contributions become dominant in the pion production in the $\gamma p$ and $\gamma \gamma$ collisions [ए7]. We point out that all the anomalous processes demand the $k_{T}$ factorization because of the end-point singularity in heavyquark decays, and the considered low- $q_{T}$ spectra. All the anomalous processes involve at least three hadrons (note that the pion production in the $\gamma p$ and $\gamma \gamma$ collisions involves fewer hadrons). These common features are the necessary conditions for the Glauber gluons to appear. It was speculated that the Glauber effect becomes significant due to the huge soft cloud of a pion, which meets its role of a NG boson [ए]8].

\section{Pion-induced Drell-Yan process}

Putting the above clues together, we estimate the Glauber effect in the pion-proton Drell-Yan process. It is the real part of $\exp \left(i S_{e}\right)$, i.e., $c_{e} \equiv \cos S_{e}$, that contributes to a cross section, so the sign of $S_{e}$ does not matter. Assigning $c_{e}$ to Fig. $\mathbb{Z}(\mathrm{a})$, and neglecting the subleading Glauber effect in Fig. $\square(\mathrm{c})$, we derived $\lambda, \mu$, and $v$ for each angular structure [प्प]. We choose the typical values $\sqrt{s}=194 \mathrm{GeV}$ and $Q=8 \mathrm{GeV}[Q]$, and the parton distribution functions (PDFs) $\phi_{\pi}(x)=$ $\phi_{p}(x) \propto x(1-x)^{2}$ for estimation. The Glauber phases $S_{e}=1.1$ and 1.2 lead to the predictions for $\lambda, v$, and the deviation $1-\lambda-2 v$ in Fig. [], which agree well with the data [0]. Note that $v$ and the deviation, being proportional to $s_{1}^{2}$, diminish at $q_{T}=0$ naturally, where the Glauber effect is supposed to disappear. $\mu$ is equal to zero, when the pion and proton PDFs have exactly the same functional form, and not presented here. That is, a nonvanishing $\mu$ can serve as a measurement of the difference between the pion and proton PDFs. It is stressed that our predictions for $\lambda$ and $v$ are insensitive to the choices of PDFs.

\section{Discussion}

The discrimination between the resolutions based on the vacuum effect and the BM functions by means of different $q_{T}$ and flavor dependencies has been reviewed in [ए0]]. Compared to the former, our proposal also involves the breakdown of the standard $k_{T}$ factorization theorem, which is, however, due to the nonperturbative mechanism from the soft cloud of the pion: the Glauber gluons correlate the quark and anti-quark distributions in hadron collision. The distinction is that our resolution is flavor dependent, and able to differentiate the $p p$ and $p d$ Drell-Yan processes from the pion-induced ones. In the BM proposal the Lam-Tung relation holds in the $p p$ and $p d$ 
processes because of the small sea-quark BM functions, but ours has nothing to do with the valence or sea type of partons. We propose to discriminate the different resolutions by measuring the $p \bar{p}$ Drell-Yan process at low $Q$ and $q_{T}$, which can be done at GSI and J-PARC. Since the anti-quark in $\bar{p}$ is a valence parton, the associated BM function is not suppressed. Then one should observe the violation of the Lam-Tung relation, similar to what was observed in the pion-induced Drell-Yan processes. According to our proposal, $\bar{p}$ is not a NG boson, so the Glauber phase is not significant, and the Lam-Tung relation should be respected. It is mentioned that the lepton angular distribution in the $p \bar{p}$ Drell-Yan process has been measured by CDF at the $Z$ pole, i.e., large $Q$, [20], and that the data are consistent with the Lam-Tung relation in a wide range of $q_{T}$, from few up to around 80 GeV.

This work was supported by the National Center for Theoretical Sciences and the National Science Council of R.O.C. under the Grant No. NSC-101-2112-M-001-006-MY3.

\section{References}

[1] C.S. Lam and W.K. Tung, Phys. Rev. D 18, 2447 (1978).

[2] E. Mirkes and J. Ohnemus, Phys. Rev. D 51, 4891 (1995).

[3] E.L. Berger, J.W. Qiu, and R.A. Rodriguez-Pedraza, Phys. Lett. B 656, 74 (2007); Phys. Rev. D 76, 074006 (2007).

[4] P. Chiappetta and M. Le Bellac, Z. Phys. C 32, 521 (1986).

[5] E866, Phys. Rev. Lett. 99, 082301 (2007); Phys. Rev. Lett. 102, 182001 (2009).

[6] S. Falciano et al. [NA10 Collaboration], Z. Phys. C 31, 513 (1986).

[7] M. Guanziroli et al. [NA10 Collaboration], Z. Phys. C 37, 545 (1988).

[8] J.S. Conway et al., Phys. Rev. D 39, 92 (1989).

[9] A. Brandenburg, O. Nachtmann, and E. Mirkes, Z. Phys. C 60, 697 (1993).

[10] D. Boer, A. Brandenburg, O. Nachtmann, and A. Utermann, Eur. Phys. J. C 40, 55 (2005).

[11] D. Boer, Phys. Rev. D 60, 014012 (1999).

[12] J. Collins and J.W. Qiu, Phys. Rev. D 75, 114014 (2007).

[13] J.C. Collins, arXiv:0708.4410 [hep-ph].

[14] G.P. Lepage and S.J. Brodsky, Phys. Lett. B 87, 359 (1979); S. Nussinov and R. Shrock, Phys. Rev. D 79, 016005 (2009).

[15] C.P. Chang and H.-n. Li, Eur. Phys. J. C 71, 1687 (2011); H.-n. Li, arXiv:1009.3610 [hep-ph].

[16] S. Mishima and H.-n. Li, Phys. Rev. D 73, 114014 (2006).

[17] A.A. Bylinkin and A.A. Rostovtsev, Eur. Phys. J. C 72, 1961 (2012).

[18] H.-n. Li and S. Mishima, Phys. Rev. D 83, 034023 (2011).

[19] C.P. Chang and H.-n. Li, Phys. Lett. B 762, 262 (2013).

[20] CDF Collaboration, Phys. Rev. Lett. 106, 241801 (2011). 